\title{
UMA ANÁLISE DA DINÂMICA SETORIALDAS MICRORREGIÕES DOS ESTADOS DE ALAGOAS E SERGIPE NO PERÍODO DE 2002 -2012
}

\author{
Paulo da Silva Santos Junior
}

Francisco José Peixoto Rosário

\begin{abstract}
RESUMO - O presente trabalho tem como objetivo analisar as características, pela sua dinâmica setorial, das 26 microrregiões de Alagoas e Sergipe, entre o ano de 2002 e 2012. Para tanto, foi utilizado o método diferencial-estrutural, na modificação de Esteban-Marquillas. Assim, é possível analisar quais as regiões que exibiram os maiores ganhos (perdas) líquidos, isto é, que apresentaram Variação Líquida Total (VLT). Os resultados obtidos indicam que há uma concentração de microrregiões de dois grupos. No primeiro, encontram-se as regiões que registraram VLT positivo, em virtude dos efeitos competitivos (C); no segundo estão as regiões que registraram VLT negativo, em decorrência da Variação estrutural (E) e do efeito alocação (A). Apesar de deterem um nível competitivo (C) positivo, não exibem uma estrutura produtiva por setores dinâmicos. Outro resultado que chamou a atenção foi que, ao decompor os efeitos de Alocação (A) entre vantagens competitivas e especialização para os setores da economia (i.e. indústria, construção civil, comércio, serviços e agropecuária), constatam-se combinações variadas das microrregiões de estudo. No entanto, com predominância de regiões que exibiram vantagens competitivas não especializadas, ou seja, o nível de emprego entre o período de 2002 a 2012 cresceu mais que a média dos dois estados, o que representa vantagem competitiva, mas o seu emprego em 2002 foi menor que o seu homotético, isto é, não especializado.
\end{abstract}

Palavra-chave: Microrregiões de Alagoas e Sergipe; Dinâmica setorial; Análise diferencialestrutural;Efeito alocação.

\section{AN ANALYSIS OF THE SECTORAL DYNAMICS OF THE MICROREGIONS OF THE STATES OF ALAGOAS AND SERGIPE IN THE PERIOD $2002-2012$}

\begin{abstract}
The objective of this study is to analyze the characteristics of the 26 microregions of Alagoas and Sergipe between 2002 and 2012. The differential-structural method was used for the modification of Esteban-Marquillas. Thus, it is possible to analyze which regions showed the largest net gains (losses), that is, that presented Total Net Change (VLT). The results obtained indicate that there is a concentration of microregions of two groups. The first one is the regions that registered positive VLT due to the competitive effects (C) and the second are the regions registered negative VLT due to the Structural variation (E) and the allocation effect (A), although they have a competitive level C) positive, do not exhibit a productive structure by dynamic sectors. Another result that drew attention was the fact that the effects of Alocação (A) between competitive advantages and specialization for the sectors of the economy (ie industry, construction, commerce, services and agriculture) show different combinations of study microregions, with a predominance of regions with non-specialized competitive advantages, that is, the level of employment between 2002 and 2012 grew more than the average of the two States, which represents a competitive advantage, but its employment in 2002 was lower than the his homothetic, that is, unspecialized.
\end{abstract}

Keyword:Microregions of Alagoas and Sergipe; Sectoral dynamics; Differential-structural analysis; Allocation effect.

\section{INTRODUÇÃO}

A partir dos anos 2000, a região Nordeste passou por transformações econômicas e sociais, baseado no tripé política social, emprego e investimento, possibilitando elevação da renda populacional, escolaridade, criação e geração de emprego, diminuição da pobreza extrema e etc. (ARAÚJO, 2014). A evolução destes indicadores, apesar de ainda não serem

$\begin{array}{llllll}\text { REVISTA ECONOMIA POLÍTICA DO DESENVOLVIMENTO } & \text { Maceió - AL } & \text { V.2 N.3. } & \text { JUNHO/2015 } & \text { P.25-42 } & \text { Página-25 }\end{array}$


satisfatórios, propiciou a diminuição do hiato de desenvolvimento existente entre o Nordeste e as regiões sulinas do Brasil (MELO, 2014).

Este período de transformações econômicas e sociais é também conhecido como "O NOVO NORDESTE", o qual somente foi possível ser alcançado tendo em vista os "sucessivos pactos federativos e sociais, responsável pela afirmação nacional dos direitos do cidadão e expansão do princípio da universalização" (AMARAL FILHO, 2010).

Araújo (2014) apresenta em seu trabalho que políticas que afetaram a renda da família (exemplo, Programa Bolsa Família) e aumento da renda real (valorização do salário mínimo) tiveram impactos regionalmente diferenciados (no termo macro). Para a autora, houve maior dinamismo econômico ${ }^{1}$ na região Nordeste do que nas regiões Sul e Sudeste, fato esse que pode ser justificado pela redução da concentração da pobreza rural, distribuídos nos pequenos municípios. Nessas pequenas municipalidades, além de possibilitar cobertura social, o novo fluxo de renda também movimenta os centros comerciais. Neste contexto, os estados de Alagoas e de Sergipe, foco desse estudo, também foram beneficiados.

Com a crescente demanda de renda, houve a dinamização do consumo, que no segundo momento propiciou o investimento. Indústrias, redes de supermercados e shopping centers se instalaram na região, principalmente nas cidades médias que passaram a crescer com mais intensidade.

No primeiro momento, a trajetória do Nordeste na evolução do consumo, na renda e no emprego ao invés da produção e do investimento, foi com maior intensidade do que nas regiões consideradas prósperas. Cabe frisar que o aumento da renda das famílias foi resultado também dos programas de infraestrutura econômica e social, expansão do crédito para o consumo e crédito ao investimento, o que também engendrou, mesmo a passos lentos, no "estreitamento" de forma significativa desta lacuna do desenvolvimento entre o Nordeste e as regiões Sul e Sudeste.

Apesar deste "estreitamento", Melo (2014) aponta que ainda não está sendo possível "avistar momento em que a região passe a apresentar indicadores econômicos e sociais próximos aos da região mais prósperas". Ainda em seu trabalho, o autor reforça que, esta diminuição da disparidade, na qual também chama de "alavancagem do processo de convergência", pode ser percebida não somente pelos indicadores de renda, mais também no emprego, na produção, na saúde e escolaridade.

Partindo dessas premissas, este estudo busca observar e analisar a estrutura produtiva e as transformações competitivas das 26 microrregiões dos estados de Alagoas e Sergipe, entre 2002 a 2012, identificando se tais regiões são especializadas e produzem vantagens competitivas (efeito alocação) para as atividades setoriais. A tipologia das estruturas produtivas nas

\footnotetext{
${ }^{1}$ Refere-se à dinamização do comércio - farmácias, lojas, padarias, feiras semanais. 
microrregiões nordestinas, segundo Betarelli \& Simões (2011) contribui para as decisões dos planejadores públicos na elaboração de políticas setoriais direcionadas para a diversificação das atividades em certas microrregiões, uma vez que, permite identificar, por exemplo, se as microrregiões que apresentam problemas na sua estrutura produtiva e detêm, ao mesmo tempo, efeitos competitivos e de alocação positivos. O intervalo de tempo escolhido se justifica por se tratar de período mais recente para dados da região, sendo um período razoavelmente longo para captar as recentes mudanças na estrutura produtivas das microrregiões. $\mathrm{O}$ fator relevante que construiu pra a escolha do período de escolha o crescimento alcançado pela região Brasileiro e principalmente dos dois Estados (entendem-se Microrregiões).

Além dessa seção introdutória e da conclusão, esse trabalho se organiza por mais duas seções: a segunda seção discute como a metodologia empregada; a terceira seção é apresentado os resultados.

\section{METODOLOGIA: DIFERENCIAL ESTRUTURAL (SHIFT-SHARE)}

O método diferencial-estrutural procura descrever o crescimento econômico de uma região em termos de sua estrutura produtiva, por meio da identificação dos componentes do crescimento. Assim, ele apresenta constatações a partir da experiência e da observação para justificar a diferença setorial e regional em um determinado período.

Para Simões (2005, p.10), o método pode ser explicado da seguinte forma:

$$
\begin{aligned}
& \text { [...] shift-share consiste, basicamente, na descrição do crescimento econômico de uma } \\
& \text { região nos termos de sua estrutura produtiva. O método é composto por um conjunto de } \\
& \text { identidades - com quaisquer hipóteses de causalidade - que procuram identificar e } \\
& \text { desagregar componentes de tal crescimento, numa análise descritiva da estrutura produtiva. }
\end{aligned}
$$

De modo simplista, Brown (1969, p.6) observa que "o método estrutural-diferencial é uma identidade formada pela adição e subtração simultâneas de taxas de crescimento, as quais são agrupadas para definir os componentes [...] sendo sempre possível incluir novas variáveis ao modelo e definir outros componentes [...]”. Além do mais, o método original aponta duas razões para o crescimento do emprego numa região: 1)Variação estrutural: que representa o somatório adicional (positivo ou negativo) que determinada região poderá obter como resultante de sua composição estrutural; 2)Variação diferencial: que indica o somatório (positivo ou negativo) que a região $\mathrm{j}$ conseguirá porque a taxa de crescimento em determinado(s) setor(es) for maior (ou menor) nesta região do que na média "nacional". O efeito diferencial indica, desta forma, as (des) vantagens locacionais da região em termos globais, qualificando os múltiplos fatores específicos da região, salientando o ritmo de crescimento regional no espaço econômico global. (LODDER, 1972).

Para Haddad (1989), estas duas variações podem ser formalizadas através das seguintes relações: 
i) o crescimento do emprego regional que pode ser dividido em três componentes: variação regional, variação estrutural e variação diferencial;

ii) variação regional que é igual ao acréscimo de emprego que teria ocorrido se uma dada região crescesse à taxa de crescimento do total de emprego nacional, no mesmo período;

iii) variação estrutural no qual representa o montante adicional de emprego que uma região poderá obter como resultante de sua composição industrial;

iv) variação estrutural que indica o montante de emprego que uma determinada região porque a taxa de crescimento do emprego, em determinados setores, foi maior ou menor nesta região do que a média nacional.

Ao estabelecer distinção entre o componente estrutural e o diferencial, este método permite identificar diferentes forças que atuam no crescimento. O componente estrutural informa que, há alguns setores que crescem mais rapidamente que os demais, além de possibilitar os fatores responsáveis por estas diferentes taxas de crescimento setorial. No entanto, o crescimento regional não pode ser examinado apenas por este ângulo, pois todos os setores produtivos apresentam desempenho diferenciado entre as várias regiões.

Apesar destas características supracitadas, o método apresenta algumas limitações. Uma das principais limitações é que não considera as mudanças na estrutura de emprego das regiões durante o período observado, isto é, entre o ano base e o ano final. Pode ocorrer, contudo, que uma região especializada em setores menos dinâmicos, no período inicial, tenha modificado a sua estrutura de emprego, de tal forma que, no período final, a sua composição industrial já tenha uma predominância relativamente maior de setores dinâmicos (HADDAD, 1989).

O autor aponta que com o objetivo de eliminar estas limitações e avaliar a sua potencialidade como técnica de projeção do crescimento regional, foram publicados diversos estudos. Entre os quais se destacam os trabalhos de Stilwell (1969), Brown (1969) e EstebanMarquillas (1972), este último sendo enfatizado neste trabalho.

Assim, Esteban-Marquillas (1972, p. 251) procurando superar algumas das objeções que são levantadas contra o método e introduz o efeito alocação e competitivo ao lado dos efeitos estrutural e diferencial, para analisar os componentes do crescimento regional a fim de eliminar a influência estrutural advinda da distribuição setorial do pessoal ocupado do ano inicial no cálculo do efeito diferencial, criou o chamado emprego homotético, ou seja, o volume de pessoal ocupado que $\mathbf{o}$ setor $\mathbf{i}$ da região $\mathbf{j}$ teria se a estrutura de emprego fosse igual à do país (Nordeste).

Contudo, os componentes de crescimento regional serão definidos como: variação regional $(\mathrm{R})$, variação estrutural $(\mathrm{E})$, variação diferencial $(\mathrm{D})$, efeito competitivo $(\mathrm{C})$ e efeito alocação (A). Dessa maneira segue:

$\sum_{i} E_{i q j}^{1}-\sum_{i} E_{i j}^{0}=\sum_{i} E_{i j}^{0}\left(r_{i t}-1\right)+\sum_{i} E_{i j}^{0}\left(r_{i t}-r_{t t}\right)+\sum_{i} E_{i j}^{\prime 0}\left(r_{i j}-r_{i t}\right)+\sum_{i}\left[\left(E_{i j}^{0}-E_{i j}^{\prime 0}\right)\left(r_{i j}-r_{i t}\right)\right]$ 

VT
$\mathbf{R}$
$\mathbf{E}$
C
A

Em VT= variação total, ou seja, a diferença entre o emprego do período final e inicial na região j;

Variação Total Líquida

$$
\mathrm{VLT}=\mathrm{VT}-\mathrm{R}=\mathrm{E}+\mathrm{C}+\mathrm{A}
$$

$\mathrm{R}=$ acréscimo do emprego se a região $\mathrm{j}$ tivesse a taxa de crescimento do emprego de todas as regiões;

$r_{t t}=\left(\sum_{i} \sum_{j} E_{i j}^{1}\right) / \sum_{i} \sum_{j} E_{i j}^{0}=\mathrm{o}$ crescimento do emprego de todas as regiões;

$r_{i t}=\sum_{j} E_{i j}^{1} / \sum_{j} E_{i j}^{0}=$ a taxa de crescimento do emprego no setor i em todas as regiões

$r_{i j}=E_{i j}^{1} / E_{i j}^{0}=$ a taxa de crescimento do emprego no setor i da região j;

$E_{i j}^{\prime}=\sum_{j} E_{i j}\left(\frac{\sum_{j} E_{i j}}{\sum_{i} \sum_{j} E_{i j}}\right)=$ emprego homotético, isto é, emprego no setor se a região $\mathrm{j}$ tivesse a mesma estrutura de emprego da principal região.

Assim, o efeito alocação possibilita verificar se a região j está especializada ou não nos setores pelos quais tenha melhores vantagens comparativas (PEREIRA, 1997). Encontram-se na tabela abaixo as quatro combinações para o efeito alocação proposto por Esteban-Marquillas ao método.

Tabela 1 -Alternativas de resultados para o efeito alocação e componentes.

\begin{tabular}{lccc}
\hline \multicolumn{1}{c}{ Alternativa } & $\begin{array}{c}\text { Efeito } \\
\text { Alocação }\end{array}$ & \multicolumn{2}{c}{$\begin{array}{c}\text { Componente } \\
\text { Vantagem } \\
\text { competitiva }\end{array}$} \\
\hline Desvantagem competitiva, especializado & negativo & + & - \\
Despantagem competitiva, não especializado & positivo & - & - \\
Vantagem competitiva, não especializado & negativo & - & + \\
Vantagem competitiva, especializado & positivo & + & + \\
\hline
\end{tabular}

Fonte: Haddad (1989)

\section{BASE DE DADOS}

Este trabalho utiliza como base de dados para estudo das 188 microrregiões nordestinas o emprego efetivo por atividade econômica de 2002 e de 2012, da Relação Anual de Informações Sociais (RAIS). Quanto ao período escolhido, justifica-se pelo fato da economia nordestina ter alcançado consideráveis níveis de crescimento econômico, sendo que o lapso temporal de uma década pode ser considerado suficiente para identificar as mudanças na estrutura produtiva das microrregiões de Alagoas e Sergipe.

Quanto à utilização da base dados, a principal vantagem de se utilizar a RAIS é que permite a desagregação das informações a nível microrregional e setorial. Contudo, a RAIS 
apresenta limitações que estão associadas à omissão e/ou sonegação de informações por parte dos declarantes, em decorrência de serem os próprios que prestam a informação, bem como apenas possuir informação de empregos formais.Outra crítica que incide é que quando existem empresas com múltiplas plantas, "devendo-se levar em consideração a possibilidade da empresa lançar o registro de seus trabalhadores na microrregião em que se localiza o escritório central e não na localização espacial efetiva do empregado" (PERREIRA, 2002).

\section{RESULTADOS E DISCUSSÕES}

Inicialmente, faz-se necessário uma análise descritiva das informações captadas na RAIS, a fim de apontar possíveis concentrações por grupos de atividades econômicos nas 26 microrregiões ${ }^{2}$ dos estados de Alagoas e Sergipe.

A tabela 2 apresenta as 10 microrregiões que mais se destacaram na participação de empregos efetivos nos anos de 2002 e 2012.

Tabela 2 -Participação do emprego efetivo por microrregião nos estados de Alagoas e Sergipe (\%) Fonte: Elaboração própria a partir dos dados da RAIS

\begin{tabular}{lcccccccccccc}
\hline \multirow{2}{*}{ Microrregião } & \multicolumn{2}{c}{ Indústria } & \multicolumn{2}{c}{ C. Civil } & \multicolumn{2}{c}{ Comércio } & \multicolumn{2}{c}{ Serviços } & \multicolumn{2}{c}{ Agropecuária } & \multicolumn{2}{c}{ Total } \\
& $\mathbf{2 0 0 2}$ & $\mathbf{2 0 1 2}$ & $\mathbf{2 0 0 2}$ & $\mathbf{2 0 1 2}$ & $\mathbf{2 0 0 2}$ & $\mathbf{2 0 1 2}$ & $\mathbf{2 0 0 2}$ & $\mathbf{2 0 1 2}$ & $\mathbf{2 0 0 2}$ & $\mathbf{2 0 1 2}$ & $\mathbf{2 0 0 2}$ & $\mathbf{2 0 1 2}$ \\
& 0,20 & 0,21 & 0,35 & 0,48 & 0,39 & 0,36 & 0,34 & 0,35 & 0,18 & 0,08 & 0,31 & 0,33 \\
Maceió(AL) & 0,14 & 0,14 & 0,53 & 0,37 & 0,33 & 0,29 & 0,35 & 0,33 & 0,09 & 0,10 & 0,30 & 0,28 \\
Aracaju (SE) & 0,24 & 0,19 & 0,02 & 0,01 & 0,01 & 0,02 & 0,03 & 0,03 & 0,23 & 0,08 & 0,08 & 0,06 \\
S. M. Campos (AL) & 0,16 & 0,13 & 0,01 & 0,01 & 0,01 & 0,02 & 0,03 & 0,03 & 0,10 & 0,11 & 0,05 & 0,05 \\
M. Alagoana (AL) & 0,03 & 0,03 & 0,01 & 0,01 & 0,07 & 0,10 & 0,04 & 0,04 & 0,03 & 0,05 & 0,04 & 0,05 \\
Arapiraca (AL) & 0,03 & 0,03 & 0,02 & 0,03 & 0,00 & 0,01 & 0,02 & 0,02 & 0,02 & 0,10 & 0,02 & 0,02 \\
B.Cotinguiba (SE) & 0,06 & 0,04 & 0,00 & 0,01 & 0,01 & 0,01 & 0,01 & 0,01 & 0,03 & 0,05 & 0,02 & 0,02 \\
S. Quilombos (AL) & 0,01 & 0,02 & 0,01 & 0,01 & 0,03 & 0,03 & 0,01 & 0,01 & 0,02 & 0,02 & 0,02 & 0,02 \\
A. de Itabaiana (SE) & 0,03 & 0,03 & 0,01 & 0,01 & 0,02 & 0,02 & 0,01 & 0,01 & 0,04 & 0,05 & 0,02 & 0,02 \\
Estância (SE) & 0,03 & 0,03 & 0,00 & 0,01 & 0,01 & 0,01 & 0,01 & 0,01 & 0,08 & 0,01 & 0,02 & 0,02 \\
Penedo (AL) & 0,08 & 0,14 & 0,03 & 0,05 & 0,11 & 0,13 & 0,16 & 0,16 & 0,16 & 0,35 & 0,13 & 0,14 \\
Demais Microrregião & $\mathbf{1 , 0 0}$ & $\mathbf{1 , 0 0}$ & $\mathbf{1 , 0 0}$ & $\mathbf{1 , 0 0}$ & $\mathbf{1 , 0 0}$ & $\mathbf{1 , 0 0}$ & $\mathbf{1 , 0 0}$ & $\mathbf{1 , 0 0}$ & $\mathbf{1 , 0 0}$ & $\mathbf{1 , 0 0}$ & $\mathbf{1 , 0 0}$ & $\mathbf{1 , 0 0}$ \\
Total & & & & & & & & & & &
\end{tabular}

Diante das informações, pode-se observar que as microrregiões Maceió e Aracaju, foram responsáveis por $61 \%$ de participação efetiva tanto nos dois períodos de análise. Em números reais, do total de 551.215 de emprego, 334.4852 foram concentrados por Maceió e Aracaju no período de 2002. Já em 2012, de 893.769 empregos, as duas microrregiões foram responsáveis por 546.405.

Segundo dados Instituto Brasileiro de Geografia e Estatística - IBGE (2016), as duas microrregiões possui um grau de urbanização de aproximadamente 100\%. Por seu turno,

\footnotetext{
${ }^{2}$ Destaca-se que as microrregiões e os setores não são desagregado, o que implica um grau maior de generalização sobre a economia do Nordeste, "uma vez que dentro das próprias microrregiões (grupo de setores), existem municípios (atividade setorial) com variado resultado de emprego e estrutura produtiva heterogênea" (BETARELLI E SIMÕES, 2010).
} 
Betarrelli e Simões (2010) afirmam que a urbanização está articulada com setor industrial e associada ao setor de serviços, setores esses que representaram em 2002 o percentual de $79 \%$ do total do emprego efetivo dos dois estados. Sendo que a indústria responde por $20 \%$ e o de serviços por 59\%. Em 2012, os dois setores representam 74\% (19\% da indústria e 55\% do serviço), apesar da redução,manteve-se o cenário de concentração.

Quando se considera apenas soma do total de empregos da indústria e serviços, é de observar que as microrregiões de Maceió e Aracaju foram responsáveis em 2002 e em 2012 por 59\% do emprego efetivo. Ou seja, apenas duas microrregiões concentram mais da metade de emprego efetivo do setor industrial e do setor de serviços. Uma explicação plausível para esta concentração reside no fato de que estas microrregiões possuem um "alto" grau de urbanização, tiveram grandes investimentos na infraestrutura no decorrer do tempo, massas de trabalhadores "qualificados", e um grande mercado consumidor.

O setor agropecuário que representa $4 \%$ do emprego nordestino em 2002 passa para $2 \%$ em 2012. As microrregiões que mais se destacaram nesse setor, foram Cotinguiba ${ }^{3}$, Mata Alagoana, Baixo Cotinguiba ${ }^{4}$, Aracaju, Maceió e São Miguel dos Campos representado 66\% na da distribuição do emprego em 2002 e $61 \%$ em 2012. O baixo desenho da agropecuária pode ser explicado pelo processo de especialização e mecanização agrícola que reduz os postos de trabalho no campo. Além do mais, outro ponto que chama a atenção é a presença das microrregiões de Aracaju e Maceió, pois como já explanado anteriormente são microrregiões um alto grau de urbanização. Esta situação pode ser explicada, tendo em vista que muitos empregadores do setor agropecuário estarem situados (registrados) nestas duas microrregiões, no entanto tem atuação em outras regiões.

$\mathrm{Na}$ contramão da indústria, serviço e agropecuária, que diminuíram a suas participações, os setores da construção civil e o comércio cresceram na participação no emprego efetivo, sendo que o primeiro passou de 4\% em 2002 para 7\% em 2012 e o segundo de $12 \%$ para $17 \%$. O bom desempenho do comércio pode ser justificado pelas políticas sociais do governo federal que estimularam o crescimento da renda das famílias, valorizaram do salário mínimo e política de crédito ativa (Araujo, 2014).

Quanto à construção civil, os investimentos na infraestrutura econômica e social possibilitaram seu desempenho (como por exemplo, o Programa de Aceleração do Crescimento - PAC ${ }^{5}$ e Programa Minha Casa Minha Vida). Nota-se que, novamente as duas microrregiões se destacaram foram Maceió

\footnotetext{
3 As microrregiões de Cotinguiba e Baixo Cotinguiba está representada nas Demais Microrregiões (Tabela 2), pois não representa nem 3\% do total do emprego efetivo nos ano 2012. Apesar deste cenário, as regiões se destacaram no setor Agropecuário de 2012, sendo que o primeiro alcançoude mais de 500\% (em 2002 tinha 3\% de participação efetiva e em 2012 passou pra 14\%) e o segundo obteve um crescimento de aproximadamente 400\% (saiu de $2 \%$ em 2002 para $10 \%$ em 2012). ${ }^{4}$ Ver nota de rodapé anterior.

${ }^{5}$ É um plano do governo federal que visa estimular o crescimento da economia brasileira, através do investimento em obras de infraestrutura (portos, rodovias, aeroportos, redes de esgoto, geração de energia, hidrovias, ferrovias, etc).
} 
e Aracaju que saíram, de 88\% de participação efetiva em 2002 para $85 \%$ em 2012. Este resultado demonstra que, apesar da redução em participação efetiva das duas microrregiões, ainda são regiões que canalizam (recebem) grande parte dos investimentos em infraestrutura.

Em síntese, conforme dados apresentados na tabela 2, as participações das microrregiões sobre o emprego efetivo total dos Estados de alagoas e Sergipe apontam poucas mudanças significativas no período de 2002 a 2012, tendo em vista que o emprego continuou concentrado em apenas duas microrregiões (Maceió e Aracaju). Ao mesmo tempo, verifica-se a perda de participação efetiva total dos setores da indústria, serviço e agropecuária, com ganhos para construção civil e comércio. Para obter melhor análise dos setores entre os períodos, é interessante analisar a medidas de concentração Coeficiente de Redistribuição (CR) (BETARELLI e SIMÕES, 2010):

\footnotetext{
Coeficiente de Redistribuição $(\mathrm{CR})^{6}$ considerada medida de localização, relaciona a distribuição percentual do emprego de um mesmo setor em dois períodos de tempo, com o objetivo de examinar se está prevalecendo para o setor algum padrão de concentração ou dispersão espacial ao longo do tempo. O seu valor oscila entre os limites 0 a 1 . Caso seja próximo de 0 não terá ocorrido mudanças significativas e caso seja próximo de 1 terá ocorrido (HADDAD, 1989).$$
C R_{i}=\sum_{j}\left(\left|j_{e i}^{t=1}-j_{e i}^{t=0}\right|\right) / 2 \text {. }
$$

Ao se calcular o CR, observou-se que o setor industrial $(0,1)$, construção civil $(0,14)$, comércio $(0,08)$, serviços $(0,06)$ e a agropecuário $(0,3)$ apresentaram coeficiente próximo de zero, ou seja, apresentaram padrão espacial com quase nenhuma mudança entre o período de 2002 a 2012. Assim, pode-se afirmar que os setores que eram concentrados em 2002, permaneceram em 2012, portanto, a concentração do emprego mostra o travamento da redistribuição das atividades setoriais pela amplitude espacial nordestina, processo que está em consonância com a "permanência" da concentração do emprego.

Neste sentido, a próxima seção, buscará captar os efeitos de alocação (vantagens competitivas e especialização) indicando quais as regiões que cresceram mais (dinâmicas) e quais cresceram menos (não dinâmicas) que a média global (nordestina), isto é, a dinâmica setorial.

\section{TIPOLOGIA DIFERENCIAL-ESTRUTURAL DAS MICRORREGIÕES}

A tipologia diferencial-estrutural procura caracterizar as microrregiões de estudo em termos de suas estruturas produtivas. Assim, para a caracterização primeiramente se considera as variações da abordagem de Esteban-Marquillas - Variação Líquida Total (VLT), Variação Estrutural (E), Efeito Competitivo (C) e Efeito de Alocação (A). Em seguida, para caracterizar as atividades setoriais, analisam-se os dois componentes do Efeito de Alocação (A) Componente de Especialização e o Componente de Vantagem Competitiva.

${ }^{6} C R_{i}=\sum_{j}\left(\left|j_{e i}^{t=1}-j_{e i}^{t=0}\right|\right) / 2$, ver em Haddad (1989).

\begin{tabular}{llllll}
\hline REVISTA ECONOMIA POLÍTICA DO DESENVOLVIMENTO & Maceió - AL & V.2 N.3. & JUNHO/2015 & P.25-42 & Página-32
\end{tabular}


Conforme visto anteriormente, a Variação Líquida Total (VLT) é um resultado combinado da Variação Estrutural (E), do Efeito Competitivo (C) e Efeito Alocação (A). Assim, Simões \& Melo (1998) expõe que a VLT indica quais as microrregiões que cresceram mais e quais cresceram menos que a média da região Nordeste. A variação Estrutural (E) indica que uma região ganhou ou perdeu em virtude de estar especializada em setores dinâmicos ou não dinâmicos. O efeito competitivo (C) capta o grau de especialização do emprego na região, na qual o mesmo está interligado com a variação estrutural e o componente diferencial da região. Finalmente, o Efeito de Alocação (A) aponta que o crescimento regional pode ocorrer porque a região detém e combina as suas vantagens competitivas com sua especialização (transformações na variação competitiva).

A partir das especificações das Variações, elaborou-se a tipologia das 26 microrregiões para o período de 2002 e 2012. Esta tipologia combina sete resultados possíveis para VLT positivo e sete para VLT negativo, no entanto somente são abordados e exibidos os que abrangem as microrregiões do Nordeste (5 VLT positivo e 5 VLT negativos). A Tabela 3 e a Figura 1 abaixo mostram as tipologias obtidas.

Tabela 3 - Tipologia das microrregiões pelo método diferencial-estrutural

\begin{tabular}{|c|c|c|c|}
\hline Cod. & $\begin{array}{c}\text { VLT } \\
\text { (SINAL) }\end{array}$ & Tipologia & Microrregião \\
\hline $\mathrm{P} 1$ & + & $\mathrm{E}, \mathrm{C}, \mathrm{A}$ positivas & Batalha (4), Arapiraca(6) e Agreste de Itabaiana(17) \\
\hline $\mathrm{P} 2$ & + & $\begin{array}{c}\text { E e C positivas } \\
\text { superam A Negativa }\end{array}$ & Maceió (11) \\
\hline P3 & + & $\begin{array}{c}\text { C positiva supera } \mathrm{E} \text { e } \\
\text { A negativas }\end{array}$ & $\begin{array}{l}\text { Serrana do Sertão Alagoano (1), Alagoana do Sertão do São } \\
\text { Francisco (2),Santana do Ipanema(3), Palmeira dos Índios(5), } \\
\text { Litoral Norte Alagoano(10), Sergipana do Sertão do São } \\
\text { Francisco(14), Carira(15), Nossa Senhora das Dores (16) e } \\
\text { Baixo Cotinguiba (23) }\end{array}$ \\
\hline $\mathrm{P} 4$ & + & $\begin{array}{c}\text { C e A positivas } \\
\text { superam E negativa }\end{array}$ & Cotinguiba (21) \\
\hline P5 & + & $\begin{array}{c}\text { A positiva supera } \mathrm{E} \\
\text { eC Negativo }\end{array}$ & Boquim (25) \\
\hline N1 & - & $\begin{array}{c}\text { E eA negativas } \\
\text { superam C positivos }\end{array}$ & $\begin{array}{l}\text { Traipu (7), Serrana dos Quilombos (8), Mata Alagoana (9), } \\
\text { São Miguel dos Campos(12), Penedo(13), Propriá(20) e } \\
\text { Estância(26) }\end{array}$ \\
\hline $\mathrm{N} 2$ & - & $\begin{array}{c}\text { A negativa supera } \mathrm{E} \\
\text { e C positivas }\end{array}$ & Tobias Barreto (18) \\
\hline N3 & - & $\begin{array}{c}\text { E eC negativas } \\
\text { superam A positiva }\end{array}$ & Japaratuba (19) \\
\hline N4 & - & $\begin{array}{c}\text { C negativo supera } E \\
\text { e A positivas }\end{array}$ & Agreste de Lagarto (22) \\
\hline N5 & - & $\begin{array}{c}\text { C e A negativas } \\
\text { superam E positiva }\end{array}$ & Aracaju (24) \\
\hline
\end{tabular}

Fonte: Elaboração própria a partir dos dados da RAIS 
Inicialmente, se observa que há uma concentração de microrregiões em dois Grupos: P3 e N1. O Grupo P3 registraram VLT positivas em virtude dos efeitos competitivos (C). Já o Grupo N1 apresentam VLT negativas em razão da variação estrutural (E) e efeito alocação (A) negativa.

Figura 1: Tipologia das microrregiões pelo método diferencial-estrutural

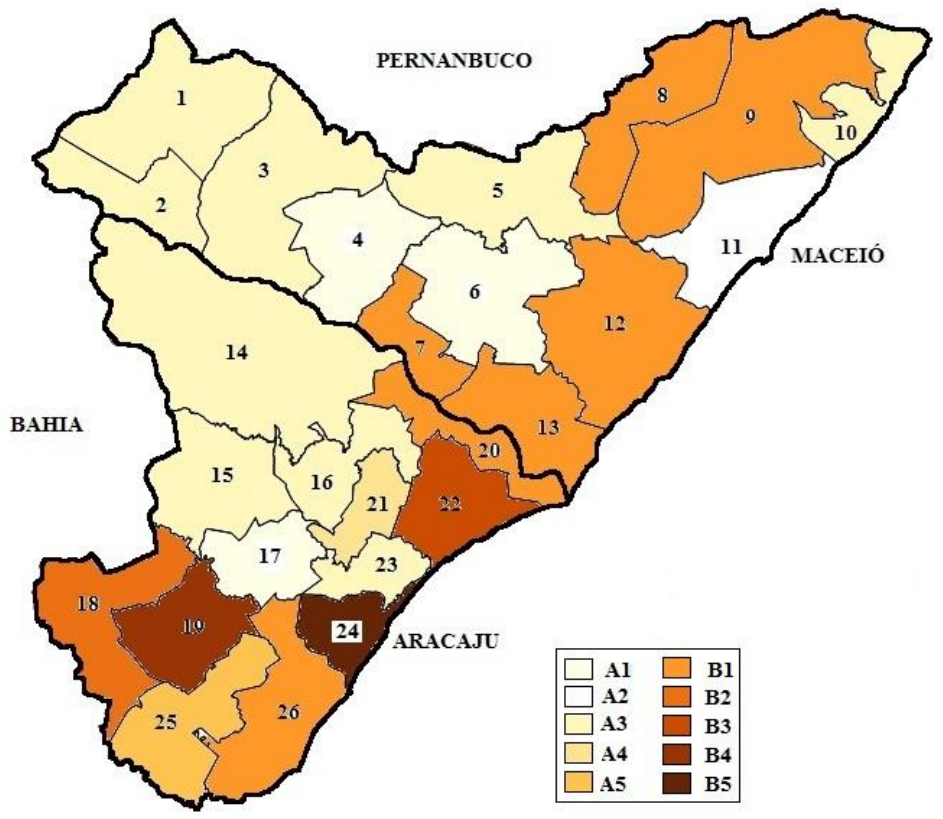

Como se observa acima que no Grupo P3 situa-se as microrregiões de Serrana do Sertão Alagoano (1), Alagoana do Sertão do São Francisco (2),Santana do Ipanema(3), Palmeira dos Índios(5), Litoral Norte Alagoano(10), Sergipana do Sertão do São Francisco(14), Carira(15), Nossa Senhora das Dores (16) e Baixo Cotinguiba (23) que apesar de exibirem efeitos de alocação (A) e não apresentarem uma estrutura produtiva por setores dinâmicos $(\mathrm{E})$, possuem um nível competitivo $(\mathrm{C})$ positivos. Com destaque para as microrregiões de Alagoana do Sertão do São Francisco e Sergipana do Sertão do São Francisco onde está localizada a hidroelétrica de Xingó, uma das maiores do Nordeste, como também destacam-se as regiões de Palmeiras dos Índios (grande produtor de leite, contanto com indústrias de laticínio, de transformação e de cana-de-açúcar; apresenta jazidas de cal, mármore, ferro e cristal de rocha) e Baixo Cotinguiba (Petrobrás - fábrica de fertilizantes e nitrogenados, Votorantim - fábrica de cimento, e a Usina São José do Pinheiro, produtora de álcool e açúcar são à base da economia).

No grupo N1 encontram-se as regiões de Traipu (7), Serrana dos Quilombos (8), Mata Alagoana (9), São Miguel dos Campos (12), Penedo (13), Propriá (20) e Estância (26), registraram VLT negativo em decorrência da Variação estrutural (E) e efeito alocação (A). Apesar de deterem um nível competitivo (C) positivo, não exibem uma estrutura produtiva por setores dinâmicos. São regiões que tem presença de indústria tradicional, por exemplo, Serrana dos Quilombos, Mata 
Alagoana, São Miguel dos Campos e Penedo tem presença forte de usina de açúcar e/ou álcool. A região de Estância destaca-se pelas indústrias alimentícias, têxteis e sucos.

Outros três grupos chamam a atenção: o P1 e P2 com VLT positivo e o grupo N5 com VLT negativo. O grupo P1 estão as regiões de Batalha (4), Arapiraca (6) e Agreste de Itabaiana (17). Em linhas gerais, isto significa que a estrutura produtiva destas microrregiões entre o período de 2002 a 2012 tornou-se ou manteve-se especializada em setores dinâmicos (E positivo), ou seja, setor da construção civil e do comércio. O efeito alocação (A) na economia das microrregiões foi positivo.

Quanto o grupo P2 e o N5 destacam-se pela presença das microrregiões de Maceió e Aracaju, respectivamente. A microrregião de Maceió, que apesar de exibir efeitos alocação (A) negativo, apresenta uma estrutura produtiva por setores dinâmicos (E) e possui um nível competitivo (C) positivo. Tanto a estrutura produtiva quanto a nível competitivo contribuiu para uma VLT positiva. No geral, a força de trabalho qualificado e especializa e infraestrutura econômica e um mercado consumidor amplo, contribuem para que as variações de $\mathrm{C}$ e $\mathrm{E}$ sejam positivas.

A região de Aracaju apresenta o VLT negativo em virtude do efeito competitivo (C) e efeito alocação (A) ser negativa. O que pode ter acontecido é que esta região perdeu dinamismo, permitindo que a indústria, por exemplo, saia da microrregião e se instale em regiões entorno e/ou interior.

Ao se decompor os efeitos de Alocação (A) entre vantagem competitiva e vantagem de especialização para todos os setores, constata-se que as combinações são variadas entre as microrregiões ${ }^{7}$. Na Figura 2 observa-se que as regiões que são especializadas e exibem vantagens competitivas para a indústria foram: Penedo (13), Agreste de Itabaiana (17), Agreste de Lagarto (22) e Boquim(25).

Figura 2: Efeito alocação desagregado para a Indústria.

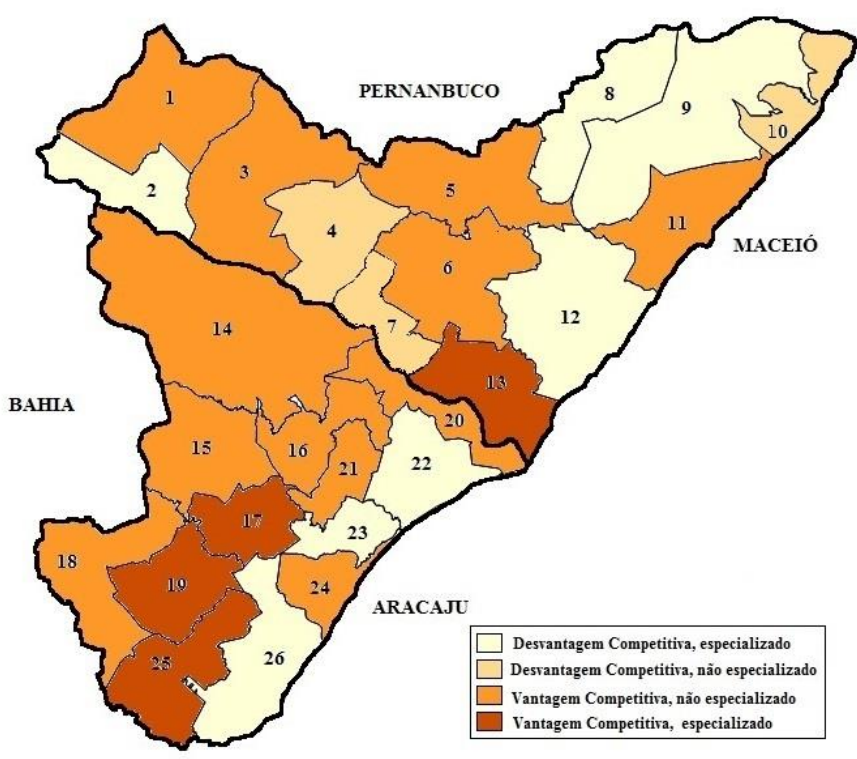

\footnotetext{
${ }^{7}$ A relação das microrregiões está descrita no apêndice.
} 
Como se observa, há poucas microrregiões que apresentam vantagens competitivas e são especializadas. No entanto, verifica-se que, são regiões que possuem características semelhantes, como por exemplo, indústrias de grande, médio e pequeno porte, além de importantesrodovias para o escoamento da produção.

Ressalta-se que as microrregiões de Agreste de Itabaiana e Boquim além de serem especialista e exibirem vantagens competitivas no setor industrial, também apresentam vantagens competitivas e são especializadas no setor agropecuário, conforme figura 3.

Figura 3: Efeito alocação desagregado para o setor Agropecuário

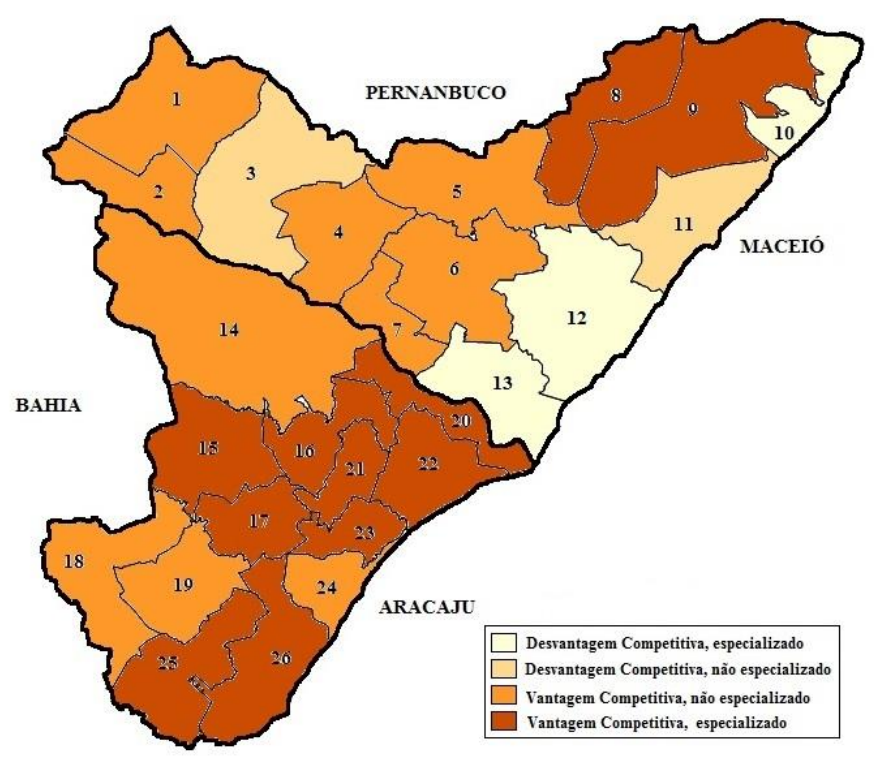

Conforme se observa na Figura 3, há uma grande concentração nas microrregiões do estado de Sergipe que apresentaram efeito alocação positivo (vantagem competitiva, especializado). No geral, são regiões que apresentam diversidade de culturas. Boquim, por exemplo, destaca-se pela citricultura (produção de laranja, tangerina, limão, além de maracujá). Ao todo, 11 microrregiões exibem vantagens competitivas e são especializadas e que na sua grande maioria abastecem as maiores regiões.

Nota-se que, assim como o setor industrial que exibe 11 microrregiões com efeito de alocação negativo (vantagem competitiva, não especializado), o setor agropecuário apresenta 10 regiões. Isso porque o nível de emprego entre o período de 2002 a 2012 cresceu mais que a média de Alagoas e Sergipe o que representa vantagem competitiva, mas o seu emprego em 2002 foi menor que o seu homotético, isto é, não especializado.

No setor de serviços (Figura 4), 17 microrregiões apresentaram vantagens competitivas. No entanto, apenas 9 regiões são especializadas o que permite a geração de um efeito de alocação (A) 
positivo. As regiões que apresentaram efeito de alocação (A) positivo foram: Serrana do Sertão Alagoano, Alagoana do Sertão do São Francisco, Santana do Ipanema, Palmeira dos Índios, Litoral Norte Alagoano, Maceió, Sergipana do Sertão do São Francisco, Tobias Barreto e Cotinguiba.

Figura 4 - Efeito alocação desagregado para o setor de Serviços

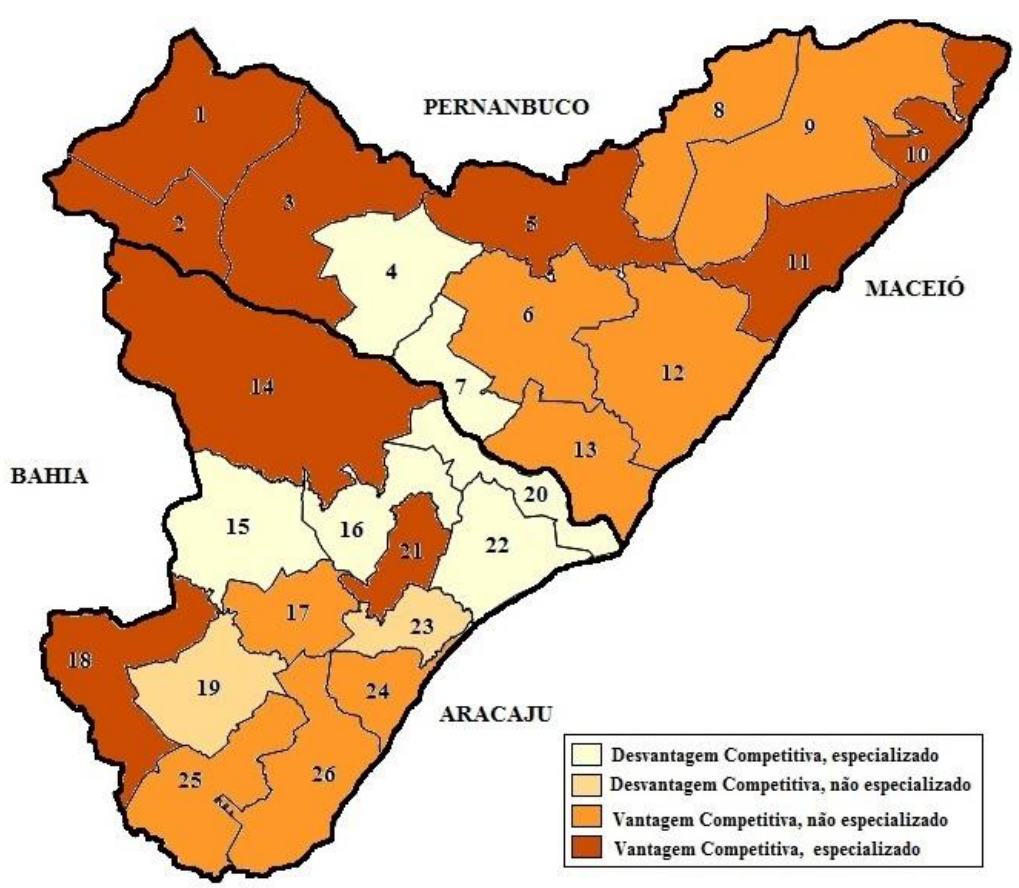

Por fim, nos setores da construção civil e comércio exibem que a grande maioria das microrregiões não é especializada e apresentam vantagens competitivas (efeito alocação negativo).

Figura 5: Efeito alocação desagregado para o setor da Construção Civil

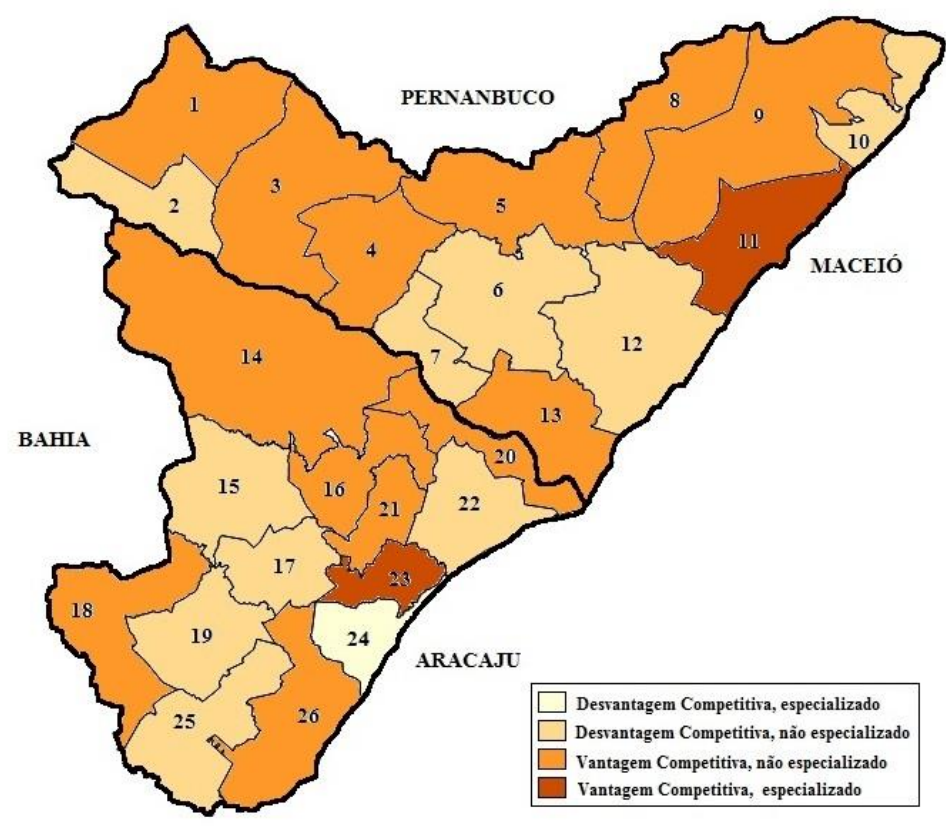


Figura 6: Efeito alocação desagregado para o setor do Comércio.

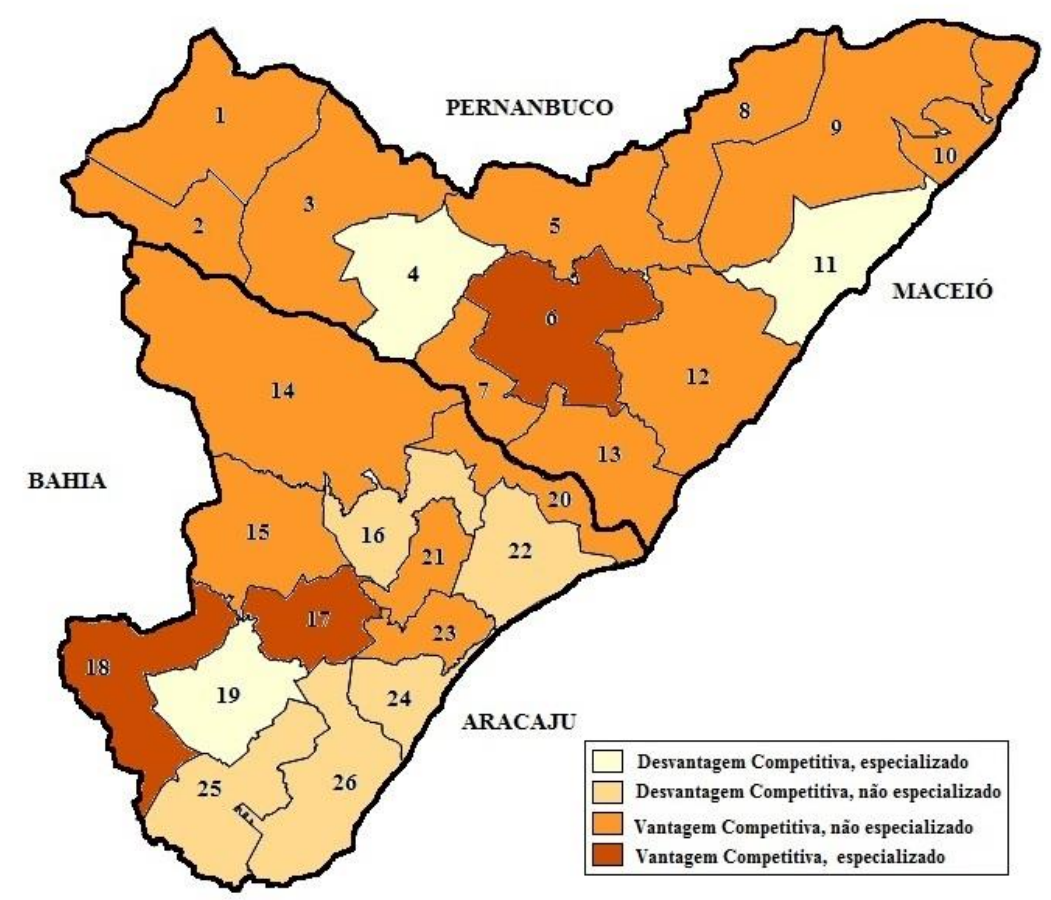

Na Figura 5 (efeito alocação desagregado da Construção Civil), nota-se também que há um grande número de regiões que apresentaram a efeito alocação positivo. Isto porque o nível de emprego entre o período de estudo (2002 a 2012) desse setor cresceu menos que a média do Nordeste (desvantagem competitiva) e porque seu emprego em 2002 foi menor que o seu emprego homotético (não especializado).

Em linhas gerais, observa-se que das 26 microrregiões, 15 apresentaram uma Variação Líquida Total positiva, quando se analisa do período de 2002 a 2012. Das regiões que exibiram ganho líquido positivo, apenas a Batalha, Arapiraca e Agreste de Itabaiana apresentaram a variação estrutural (E), o efeito competitivo $(C)$ e o efeito alocação (A) positivos. Tais regiões especializadas e com vantagem competitiva nos seguintes setores:

- A região do Agreste de Itabaiana é especializada e exibe vantagens comparativas na indústria, comércio e agropecuária;

- Já a microrregião de Arapiraca, apresenta no setor do comércio;

Desse grupo, a única microrregião que não apresentou especialização e vantagem competitiva em nenhum setor foi Batalha. Por outro lado, quando se analisa o efeito alocação por setorial de todas as regiões, verificou-se o seguinte resultado: em todos os setores, há maiores concentrações de regiões não especializadas e com vantagens comparativas. No setor industrial, localiza-se 11 regiões; na agropecuária exibe 10; construção civil, 13; e o comércio 15 e no setor de Serviços, 8. Ressalta-se que, a construção civil também apresenta um bom 
grupo regiões não especializadas com desvantagem competitiva, 10 microrregiões. Por fim, o setor de Serviços e Agropecuário também se destaca o grupo regiões que exibiram especializadas com vantagem competitiva, totalizando 9 para o primeiro e 11 para o segundo.

\section{CONCLUSÃO}

Este trabalho objetivou entre outros pontos, oferecer contribuições acerca das características das 26 microrregiões de Alagoas e Sergipe por meio da dinâmica de crescimento do emprego no período de 2002 e 2012 utilizando como metodologia o método diferencialestrutural na modificação de Esteban-Marquillas.

Para obter melhor análise dos setores entre os períodos, buscou-se mostrar inicialmente se estava prevalecendo algum padrão de concentração ou dispersão espacial ao longo do tempo (Coeficiente de Redistribuição). No primeiro, o resultado mostrou que os setores que eram concentrados em 2002 se mantiveram em 2012.

$\mathrm{Na}$ tipologia diferencial-estrutural, que caracteriza as microrregiões em termos de sua estrutura produtiva, o resultado mostrou concentração de microrregiões em dois grupos: o primeiro (P3, concentra 9 microrregiões) exibe as regiões que registraram ganhos líquidos positivos, em razão dos efeitos competitivos. Com destaques para Palmeiras dos Índios (grande produtor de leite contanto com indústrias de laticínio, de transformação e de cana de açúcar;) e Baixo Cotinguiba (Petrobrás - fábrica de fertilizantes e nitrogenados, Votorantim - fábrica de cimento, e a Usina São José do Pinheiro, produtora de álcool e açúcar são à base da economia). O segundo grupo (N1, concentra 7) aponta as regiões que registraram VLT negativo em decorrência da variação estrutural e efeito alocação, isto é, apresentam nível competitivo positivo, mas não exibem uma estrutura produtiva por setores dinâmicos.

Quanto aos grupos P1, P2 (VLT positivo), destacasse: no P1 exibe as regiões que exibiram a estrutura produtiva destas microrregiões entre o período de 2002 a 2012 tornou-se ou manteve-se especializados em setores dinâmicos, bem como o efeito alocação e vantagem competitiva positiva. Situam-se as microrregiões de Batalha, Arapiraca e Agreste de ItabaianaSE; no P2 está Maceió, região de que apesar de exibir efeito alocação negativo, apresenta uma estrutura produtiva por setores dinâmicos e possuem um nível competitivo positivo. No geral, força de trabalho qualificado e especializada em infraestrutura econômica e um mercado consumidor amplo, contribuem para que as variações de $\mathrm{C}$ e $\mathrm{E}$ sejam positivas.

Quanto aos grupos que exibem ganhos líquidos negativos (VLT negativo), destacasse o N6 onde está a microrregião de Aracaju, vez que apresentam o VLT negativo em virtude do efeito competitivo e efeito alocação serem negativos. 
Ao decompor o efeito alocação, entre vantagem competitiva e de especialização se constatou que nos setores analizados, há grandes concentrações de regiões em que o nível de emprego entre o período de 2002 a 2012 cresceu mais que a média de alagoas e Sergipe (juntos) o que representa vantagem competitiva, mas o seu emprego em 2002 foi menor que o seu homotético, isto é, não especializado.

Assim, esse trabalho procurou auxiliar na caracterização da economia de Alagoas e Sergipe no período de 10 anos, apontando as microrregiões que possuem um dinamismo setorial diferenciado.

\section{REFERÊNCIA BIBLIOGRÁFICAS}

AMARAL FILHO, J. A endogeneização no desenvolvimento econômico regional e local. Planejamento e Políticas Públicas (IPEA), v. 23, 2001p. 261-286.

O Nordeste que dá certo. Cadernos do Desenvolvimento: Centro Internacional Celso Furtado de Políticas para o Desenvolvimento. Rio de Janeiro, v. 05, 2010p. 53-83.

ARAUJO, T. B. Nordeste: desenvolvimento recente e perspectivas. In: GUIMARÃES, P. F. et al (Org). Um olhar territorial para o desenvolvimento: Nordeste. Rio de Janeiro: BNDES, 2014, p. 540-562.

BETARELli JUNIOR, A.; SIMÕES, R. A Dinâmica Setorial e os Determinantes Locacionais das Microrregiões Paulista. Economia Aplicada. São Paulo, v. 15, n.4, p. 641-670, out./dez 2011.

BROWN, H. J. Shift and share projections of regional economic growth: and empirical test. Journal of Regional Science, v.9, n.1, p.1-17, 1969.

HADDAD, P. R. Economia regional, teorias e métodos de análise. Technical report, Fortaleza: BNB, 1989.

LODDER, C. Crescimento da ocupação regional e seus componentes. In HADDAD, P. R. Planejamento regional: método e aplicação ao caso brasileiro. Rio de Janeiro: IPEA/INPES, 1972 (Série Monográfica, 8).

MELO, R. O. L. Uma janela de oportunidade para o Nordeste.In: GUIMARÃES, P. F. et al (Org). Um olhar territorial para o desenvolvimento: Nordeste. Rio de Janeiro: BNDES, 2014, p. 464-501.

PEREIRA, A. S. O método estrutural-diferencial e suas reformulações. Teor. Evid. Econ. Passo Fundo, v. 5, n.9, p. 91-103, maio 1997.

PEREIRA, F. M. Cidades médias brasileiras: uma tipologia a partir de suas (des) economias de aglomeração. Master'sthesis, Universidade Federal de Minas Gerais (CEDEPLAR/UFMG), 2002.

SIMÕES, R.Métodos de Análise Regional e Urbana: diagnóstico aplicado ao planejamento. Texto para Discussão, n. 259. Belo Horizonte: Cedeplar/UFMG, 2005. 


\section{APÊNDICE}

ATipologia dos resultados do método diferencial-estrutural 
Tabela A.1 - Tipologia dos resultados do método diferencial-estrutural

\begin{tabular}{|c|c|c|c|c|c|c|c|}
\hline Cod. & Microrregião & Classe & Indústria & C. Civil. & Serviços & Comércio & Agropecuário \\
\hline 1 & Serrana do Sertão Alagoano & P3 & V. Comp. não esp. & V. Comp. não esp. & V. Comp., esp. & V. Comp. não esp. & V. Comp. não esp. \\
\hline 2 & Alag. do Sertão do S. Franc. & $\mathrm{P} 3$ & Desv. Comp., esp. & Desv. Comp.,não esp. & V. Comp., esp. & V. Comp. não esp. & V. Comp. não esp. \\
\hline 3 & Santana do Ipanema & P3 & V. Comp. não esp. & V. Comp. não esp. & V. Comp., esp. & V. Comp. não esp. & Desv. Comp.,não esp. \\
\hline 4 & Batalha & $\mathrm{P} 1$ & Desv. Comp.,não esp. & V. Comp. não esp. & Desv. Comp., esp. & Desv. Comp., esp. & V. Comp. não esp. \\
\hline 5 & Palmeira dos Índios & $\mathrm{P} 3$ & V. Comp. não esp. & V. Comp. não esp. & V. Comp., esp. & V. Comp. não esp. & V. Comp. não esp. \\
\hline 6 & Arapiraca & $\mathrm{P} 1$ & V. Comp. não esp. & Desv. Comp.,não esp. & V. Comp. não esp. & V. Comp., esp. & V. Comp. não esp. \\
\hline 7 & Traipu & $\mathrm{N} 2$ & Desv. Comp.,não esp. & Desv. Comp.,não esp. & Desv. Comp., esp. & V. Comp. não esp. & V. Comp. não esp. \\
\hline 8 & Serrana dos Quilombos & N1 & Desv. Comp., esp. & V. Comp. não esp. & V. Comp. não esp. & V. Comp. não esp. & V. Comp., esp. \\
\hline 9 & Mata Alagoana & N1 & Desv. Comp., esp. & V. Comp. não esp. & V. Comp. não esp. & V. Comp. não esp. & V. Comp., esp. \\
\hline 10 & Litoral Norte Alagoano & $\mathrm{P} 3$ & Desv. Comp.,não esp. & Desv. Comp.,não esp. & V. Comp., esp. & V. Comp. não esp. & Desv. Comp., esp. \\
\hline 11 & Maceió & $\mathrm{P} 2$ & V. Comp. não esp. & V. Comp., esp. & V. Comp., esp. & Desv. Comp., esp. & Desv. Comp.,não esp. \\
\hline 12 & São Miguel dos Campos & N1 & Desv. Comp., esp. & Desv. Comp.,não esp. & V. Comp. não esp. & V. Comp. não esp. & Desv. Comp., esp. \\
\hline 13 & Penedo & N1 & V. Comp., esp. & V. Comp. não esp. & V. Comp. não esp. & V. Comp. não esp. & Desv. Comp., esp. \\
\hline 14 & Serg. do Sertão do S. Franc. & P3 & V. Comp. não esp. & V. Comp. não esp. & V. Comp., esp. & V. Comp. não esp. & V. Comp. não esp. \\
\hline 15 & Carira & P3 & V. Comp. não esp. & Desv. Comp.,não esp. & Desv. Comp., esp. & V. Comp. não esp. & V. Comp., esp. \\
\hline 16 & Nossa Senhora das Dores & $\mathrm{P} 3$ & V. Comp. não esp. & V. Comp. não esp. & Desv. Comp., esp. & Desv. Comp.,não esp. & V. Comp., esp. \\
\hline 17 & Agreste de Itabaiana & P1 & V. Comp., esp. & Desv. Comp.,não esp. & V. Comp. não esp. & V. Comp., esp. & V. Comp., esp. \\
\hline 18 & Tobias Barreto & $\mathrm{N} 2$ & V. Comp. não esp. & V. Comp. não esp. & V. Comp., esp. & V. Comp., esp. & V. Comp. não esp. \\
\hline 19 & Agreste de Lagarto & N4 & V. Comp., esp. & Desv. Comp.,não esp. & Desv. Comp.,não esp. & Desv. Comp., esp. & V. Comp. não esp. \\
\hline 20 & Propriá & N1 & V. Comp. não esp. & V. Comp. não esp. & Desv. Comp., esp. & V. Comp. não esp. & V. Comp., esp. \\
\hline 21 & Cotinguiba & $\mathrm{P} 4$ & V. Comp. não esp. & V. Comp. não esp. & V. Comp., esp. & V. Comp. não esp. & V. Comp., esp. \\
\hline 22 & Japaratuba & N3 & Desv. Comp., esp. & Desv. Comp.,não esp. & Desv. Comp., esp. & Desv. Comp.,não esp. & V. Comp., esp. \\
\hline 23 & Baixo Cotinguiba & $\mathrm{P} 3$ & Desv. Comp., esp. & V. Comp., esp. & Desv. Comp.,não esp. & V. Comp. não esp. & V. Comp., esp. \\
\hline 24 & Aracaju & N5 & V. Comp. não esp. & Desv. Comp., esp. & Desv. Comp., esp. & Desv. Comp.,não esp. & V. Comp. não esp. \\
\hline 25 & Boquim & P5 & V. Comp., esp. & Desv. Comp.,não esp. & V. Comp. não esp. & Desv. Comp.,não esp. & V. Comp., esp. \\
\hline 26 & Estância & N1 & Desv. Comp., esp. & V. Comp. não esp. & V. Comp. não esp. & Desv. Comp.,não esp. & V. Comp., esp. \\
\hline
\end{tabular}

Fonte: Elaboração Própria

\begin{tabular}{llllll}
\hline REVISTA ECONOMIA POLÍTICA DO DESENVOLVIMENTO & Maceió - AL & V.2 N.3. & JUNHO/2015 & P.25-42 & Página-42
\end{tabular}

